\title{
The international conservation importance of Welsh 'waxcap' grasslands
}

Griffith GW $^{1 *}$, Gamarra JGP ${ }^{1}$, Holden $\mathbf{E M}^{2}$, Mitchel ${ }^{3}$, Graham $A^{4}$, Evans $\mathrm{DA}^{5}$, Evans $\mathrm{SE}^{6}$, Aron $\mathrm{C}^{7}$, Noordeloos $\mathrm{ME}^{8}$, Kirk PM${ }^{9}$, Smith SLN ${ }^{10}$, Woods $\mathrm{RG}^{1}$, Hale $\mathrm{AD}^{10}$, Easton $\mathrm{GL}^{1}$, Ratkowsky $\mathrm{DA}^{11}$, Stevens $\mathrm{DP}^{10 \dagger}$ and Halbwachs $\mathrm{H}^{12}$

\footnotetext{
${ }^{1}$ Institute of Biological, Environmental and Rural Sciences, Cledwyn Building, Aberystwyth University, Penglais, Aberystwyth, CeredigionSY23 3DD (gwg@aber.ac.uk,jgg@aber.ac.uk,gre@aber.ac.uk)

${ }^{2}$ Allanaquoich, Mar Lodge Estate, Braemar, Ballater, Aberdeenshire, AB35 $5 Y J$ Scotland UK (liz@marmycology.co.uk)

${ }^{3}$ Walnut Tree Cottage, Llanthomas Lane, Llanigon, Powys HR3 5PU Wales UK (david.mitchel@nifg.org.uk)

${ }^{4}$ Trawscoed, Llanuwchllyn, Bala, Gwynedd LL23 7TD Wales UK (angrhm@globalnet.co.uk)

${ }^{5}$ Rowen, Y Felinheli, Bangor, Gwynedd LL56 4RX Wales UK (debbie.felin@btinternet.com)

${ }^{6}$ Lower Penylan, Glasbury-on-Wye, Powys HR3 5NT Wales UK (shelley.evans@mycoservices.org.uk)

${ }^{7} 4$ Refail Fields Pentraeth, Ynys Môn LL75 8YF Wales UK (AronMycology@aol.com)

${ }^{8}$ National Herbarium of the Netherlands, P.O. Box 9514, Einsteinweg 2, Leiden, Netherlands (m.noordeloos@mac.com)

${ }^{9}$ Royal Botanic Gardens, Kew Richmond, Surrey TW9 3AB, UK (p.kirk@kew.org)

${ }^{10}$ Cyfoeth Naturiol Cymru, Maes-y-Ffynnon, Penrhosgarnedd, Bangor LL57 2DW Wales UK (alan.hale@cyfoethnaturiolcymru.gov.uk; stuart.smith@cyfoethnaturiolcymru.gov.uk; ${ }^{* \dagger}$ Deceased)

${ }^{11}$ School of Agricultural Science, University of Tasmania-Hobart, Private Bag 54, Hobart, Tasmania 7001 Australia (ratkowsk@postoffice.utas.edu.au)

${ }^{12}$ Bavarian Mycological Society, D-63916 Amorbach, Germany (halb.wax@onlinehome.de)
}

Griffith GW, Gamarra JGP, Holden EM, Mitchel D, Graham A, Evans DA, Evans SE, Aron C, Noordeloos ME, Kirk PM, Smith SLN, Woods RG, Hale AD, Easton GL, Ratkowsky DA, Stevens DP, Halbwachs H 2013 - The international conservation importance of Welsh 'waxcap' grasslands. Mycosphere 4(5), 969-984, Doi 10.5943/mycosphere/4/5/10

\begin{abstract}
The large decline in plant and animal diversity of semi-natural grasslands resulting from the introduction of modern agricultural practices in the 1940's has been well documented and such changes are also suspected of causing the decline in the abundance and diversity of macrofungi in these habitats. We conducted repeated surveys at 48 selected grassland sites around Wales to record the presence and abundance of fruitbodies (FBs) of grassland macrofungi belonging to the taxa Clavariaceae, Hygrocybe, Entoloma, Geoglossaceae, Dermoloma (also Porpoloma and Camarophyllopsis spp.) which are grouped collectively as "CHEGD" fungi (acronym of group names) and considered typical of nutrient poor 'waxcap' grasslands. A total of 111 CHEGD species (of the ca. 200 species previously found in UK/Ireland) were recorded. That these included one

\footnotetext{
Abbreviations: BMS - British Mycological Society; CCW - Countryside Council for Wales; FB - fruitbodies; FRDBI - Fungal Records Database of Britain and Ireland; GIS - Geographical Information System; NVC - National Vegetation Classification; SSSI - Sites of Special Scientific Interest; VC - (Watsonian) Vice-county
} 
species unknown to science, 14 new to Wales including two new to the UK attests to the extent of past under-recording. Phenological differences in fruiting were found between Entoloma and Hygrocybe spp., and patterns of occurrence at the sites correlated well with numbers of records from the Fungal Records Database of Britain and Ireland. The recent post-glacial history and high human population densities have generally resulted in lower levels of biological diversity in northwestern Europe than in other parts of the world. Compared with current data from other European countries and globally, Welsh grasslands host particularly diverse macrofungal communities, yet the organisms continue to attract little attention from mainstream conservation bodies, whilst much conservation effort is lavished on species which are relatively common elsewhere.

Keywords - EU habitats directive - macrofungal diversity - red data list - species richness - UK biodiversity action plan (BAP)

\section{Introduction}

In terms of ecosystem function, the process of decomposition is of comparable importance to net primary production, yet the diversity of the soil biota, of which the fungi form a crucial component due to their role in lignocellulose catabolism, is seldom considered in the context of biodiversity and conservation. Technological constraints limit the study of these mainly microscopic organisms but the macrofungi, forming more readily visible fruiting structures, are more amenable to field study. Despite this, it is rare to find general biological surveys which make any mention of these organisms. In part this is due to limitations in taxonomic expertise, but an additional factor is that fruiting of these fungi usually occurs outside the usual 'field season' for ecologists. Nonetheless, macrofungal fruit bodies (FBs) provide a visible indication of the status of the soil biota, acting as a potentially useful surrogate for the diversity of other soil organisms (Bardgett \& McAlister 1999).

The past 75 years have witnessed a loss of more than $90 \%$ of unimproved grasslands in western Europe, mainly due to agricultural intensification (Lovegrove et al. 1995, Hewins et al. 2005, Stevens et al. 2009). The consequential threats to plants, insect, mammal and bird populations are well-recognised (Benton et al. 2002, Flynn et al. 2009), but effects on macrofungi in these habitats have received relatively much less consideration.

The species richness of macrofungi in woodland habitats is greater than in grasslands; however, it is estimated that about 400 species (some 10\% of the total number of species) are, in North-Western Europe, specific to, or mainly found in grasslands (Arnolds \& de Vries 1989, Arnolds 1992), with members of the genus Hygrocybe, ('waxcaps') and grassland representatives of Entolomataceae ('pink gills'), Clavariacaeae ('fairy clubs') and Geoglossaceae ('earth tongues'), as well as the genera Camarophyllopsis, Dermoloma and Porpoloma, comprising about half of this number. These fungi, which are characteristic of less disturbed, unfertilised grasslands, are sometimes referred to collectively as CHEG/CHEGD fungi (acronym of the taxon names), though it is the colourful and charismatic waxcaps (Hygrocybe spp.) that have attracted greatest attention.

Concerns about the decline of fungal diversity in grasslands were first raised by Arnolds (1982, 1988) in the Netherlands, and later Rald (1985), Nitare (1988) and Jordal (1997) in Scandinavia, with Arnolds warning that only ca. 200 ha of 'waxcap grassland' habitat remained in the Netherlands. Subsequent surveys in other parts of Europe found that these fungi predominantly occurred in undisturbed permanent pastures, and that in upland areas of the UK such habitats are relatively more abundant than in continental Europe (Rotheroe et al. 1996, Newton et al. 2003). These earlier studies established not only that the UK contains a relatively large number of sites with a high species-richness of grassland fungi but also the extent of under-recording of these taxa. Prior to the 1990s there is very limited data for distributions of grassland macrofungi, so that historical examinations can only be inferred from the loss of their typical habitats.

Grassland fungi do now have some profile within conservation planning, with $>75 \%$ of the ca. 200 CHEGD species found in UK grasslands included in the Red Data Lists (RDL) for at least one European country. Ten of these species (including eight Hygrocybe spp.) are present on the 
RDL of nine or more of the 31 countries for which data have been published (PM Kirk, pers. comm. 2009, Senn-Irlet et al. 2007)). National action programmes which include grassland fungi have also been established in several countries, for instance the UK Biodiversity Action Plan which includes four CHEGD species (http://jncc.defra.gov.uk/page-5165), with habitat-based approaches such as the establishment of Important Fungal Areas and the EU Habitat Directive now being implemented at national level in several countries to include macrofungi (Senn-Irlet et al. 2007). Of the dozen or so sites in Europe which are legally protected due to their mycological importance, five are grassland sites (four in Wales, one in England). Concurrently, research into the ecology of waxcaps and other grassland fungi aimed at informing future conservation strategies is also underway (Griffith et al. 2002, 2004).

The recording of grassland fungi, initially by pioneering mycologists such as Schaeffer, Scopoli and Wulfen in the late $18^{\text {th }} \mathrm{C}$ (in Central Europe), noted the occurrence of Hygrocybe spp. (then classified as Agaricus) in association with grasses and mosses in hay meadows (in pratis) and pastures (in pascuis), occasionally in woodland. Similarly in Western Europe, Sowerby, Bulliard, and Withering described the occurrence of the same suite of fungi in grasslands (e.g. Kensington Park, London), with Davies (1813) also reporting the occurrence of many of these fungi in Wales (e.g. A. psittacinus "and a number of beautiful varieties").

The publication of accessible keys to these taxa, notably monographs of the genera Hygrocybe (Boertmann 1995) and Entoloma (Noordeloos 1992, Vesterholt 2002, Noordeloos 2004) have provided a robust taxonomic framework, whilst the availability of national checklists of macrofungi for many countries in Europe and beyond (Senn-Irlet et al. 2007) has facilitated taxonomic consistency for most of these groups. It should nevertheless be noted that particularly for waxcaps molecular analyses have shown distinct differences between species from different ecozones and has altered the taxonomic concept of Hygrocybe (Lodge et al. 2006).

With the advent of more formal recording of macrofungi in many countries, there are now many national databases, the largest being the Fungal Records Database of Britain and Ireland (FRDBI; >1,000,000 records in total; http://www.fieldmycology.net/). About 25\% (>25,000) of the FRDBI records for CHEGD species are from Wales, the majority (>80\%) from 1998 onwards, due to increased and more formalised recording activity. Paradoxically, this recent increase in recording activity can give the totally misleading impression that the distribution and abundance of these fungi is increasing, whereas the precipitous decline in the area of unimproved grasslands in Wales (Stevens et al. 2007, 2009) and elsewhere in the UK would strongly suggest that the opposite is the case.

Interpretation of survey records can be difficult since they are generally collected in a nonstandardised manner. Whilst they show which species are present and can indicate approximate levels of abundance, the addressing of conservation priorities requires the development of more systematic approaches to identify sites of high conservation value. The ephemeral, weatherdependent and often irregular nature of macrofungal fruiting makes these aims all the more challenging to achieve. A number of previous studies have described methodologies for more quantitative assessment of fungal diversity (Cannon 1997, Feest 2000, Straatsma et al. 2001) but these have mainly been applied in woodland habitats. To our knowledge the only previous systematic regional/national surveys of grassland fungi are in Somerset (Thompson 2000) and Scotland (Newton et al. 2003). The former study conducted repeated surveys of Hygrocybe spp. at 18 grassland sites over a three year period using the survey methodology developed by Feest (2000), whilst the latter conducted (mainly) single visit surveys of more than 500 sites over a three year period.

The aim of the present study was to assess populations of grassland (CHEGD) fungi at a range of sites across Wales, providing a data set essential for conservation purposes. In contrast to the earlier work of Newton et al. (2003), our study focused on a smaller number of sites of defined area known to host diverse populations of grassland fungi, with each site being surveyed twice to determine seasonal and inter-year differences in the fungi recorded. 


\section{Methods}

Field surveys were conducted at 48 sites between 2003 and 2005, with each site being visited twice during this period, in different years. In most cases, the repeat surveys were conducted during a different period of the fruiting season (i.e. 'early' and 'late' in the different years; Table 1). Choice of the sites surveyed in the present study was guided by prior knowledge derived from either information collated by Evans \& Holden (2003), the CCW Lowland Grassland Survey (Stevens et al. 2007, 2009), input from CCW Conservation Officers and sites identified by individual surveyors. Thus the initial aim was to focus on sites known or suspected to host diverse populations of grassland fungi. In most cases the area surveyed was defined by ownership or SSSI (Sites of Special Scientific Interest) boundaries, though for 10 sites, the survey area was defined by the surveyors. In all cases, the surveyed area was recorded in GIS format.

Surveys of grassland fungi have tended to focus primarily on members of the genus Hygrocybe. It is recognized that members of several other taxa co-occur with these and here we use the acronym CHEGD fungi, modified from the Rotheroe's (1999) CHEG acronym which was based on the initials of the four main groups. There have also been other variations in the methods for categorisation and counting of these fungi and also which species are included among the target taxa. For clarity and consistency, we have followed the convention of Nitare (1988) as follows:-

C: Members of the Clavariaceae in the genera Clavaria, Clavulinopsis and Ramariopsis.

H: members of the genus Hygrocybe, sensu Boertmann (1995) [the concept for this genus differs outside Europe but all fall within the Hygrophoraceae].

E: members of the genus Entoloma sensu Noordeloos (1992, 2004), listed by Legon \& Henrici (2005) as occurring in grasslands.

G: Members of the Geoglossaceae in the genera Geoglossum, Trichoglossum (and Microglossum). This ascomycete taxon is beyond the scope of Legon (2005) and no definitive monograph currently exists; the classifications of Ridge (2006) or Silverside (1997) are followed here.

D: All species of Dermoloma and Camarophyllopsis plus Porpoloma metapodium.

Using these criteria, members of the target species (not subspecies) listed above were recorded, with taxon totals being presented as follows - C:H:E:G:D. For the basidiomycetes (all within the order Agaricales), species names follow those used by the recently published $U K$ Checklist of Basidiomycetes (Legon \& Henrici 2005). Only species listed herein as being found in grassland habitats were counted, excluding strictly woodland species found in or adjacent to wooded areas. For example, 65 of the 157 UK species of Entoloma listed by Legon \& Henrici (2005) are noted as occurring only in woodland habitats. Subspecies were recorded but did not contribute additionally to site totals.

Spatial data were handled using the MapInfo 8.0 GIS package. Data were initially handled in Excel which was also used for graph drawing. Pearson correlation analysis and tests of normality of data were conducted using Minitab (version 15).

\section{Results}

\section{Survey Sites}

The 48 sites surveyed (Fig. 1; Table 1) are located in 45 different Ordnance Survey $10 \mathrm{~km}$ grid squares including all the 13 Watsonian vice-counties (UK units for biological recording) in Wales. Most site surveys for macrofungi generate lists of species recorded during each visit but only rarely is the site explicitly defined. The FRDBI guidelines suggest that, in addition to grid references, the nearest named location on the OS map is also given, though for most submitted records the area of the site (or the area surveyed) is not stated. Since species-area arguments (Rosenzweig 1995) would suggest that larger sites should host more species (and are also more likely to encompass more grassland habitat sub-types), care was taken to explicitly define the area of all sites (Table 1). Where possible these were defined by ownership boundaries (a practical 
definition since conservation management planning would be conducted on the same basis). A range of management strategies were in place at the different sites (based on surveyor observations on the survey days), with sheep grazing (either alone or in combination with other herbivores) being the commonest form of management (35 sites), followed by rabbit grazing (11), cattle grazing (9), horse grazing (7) and mowing (6).

Sites ranged in area from 0.11 to 14,568 ha (median area 30.5 ha). Most sites consisted predominantly of grassland habitats, though there were significant proportions of other habitat types at some sites (e.g. Trawscoed [Table 1, site 22] comprises 235 ha moorland, 135 ha woodland and 35 ha mesotrophic/upland grassland) but these non-grassland habitats were not surveyed. However, even with use of GIS with data from the CCW Lowland Grassland Survey (Stevens et al. 2007,2009 ) it was not possible to quantify the percentage of grassland area for many of the sites. Since the sites varied considerably in area, it was also difficult to equalize survey effort, with factors such as site topology, weather and vegetational heterogeneity (including sward height) all influencing the rate of progress around the site and ease of discovery of macrofungi.

Based on the experiences of Newton et al. (2003), each surveyor (eight in total), all experienced mycologists familiar with surveying of grassland sites, were directed to use their intuition rather than any formalized systematic approach. For smaller sites $(<1 \mathrm{ha})$, surveys were completed in less than $2 \mathrm{hrs,} \mathrm{with} \mathrm{intermediate} \mathrm{sites} \mathrm{(}<100 \mathrm{ha}$ ) being allocated a whole day and the largest sites two days. For the larger sites (eight were $>200$ ha), where a complete survey would clearly not have been possible during the time allocated, sites were accessed from several directions to ensure that widely distributed sub-areas including different grassland habitat sub-types were surveyed. There was a significant positive correlation between CHEGD totals and $\log _{10}$ [site area] $(\mathrm{r}=0.455 ; \mathrm{p}<0.001)$. As such, the seven sites with the highest CHEGD scores were all large $(>80$ ha) and of the 16 sites covering $>80$ ha, only two (Castlemartin and Cors Geirch) had fewer than 30 CHEGD species.

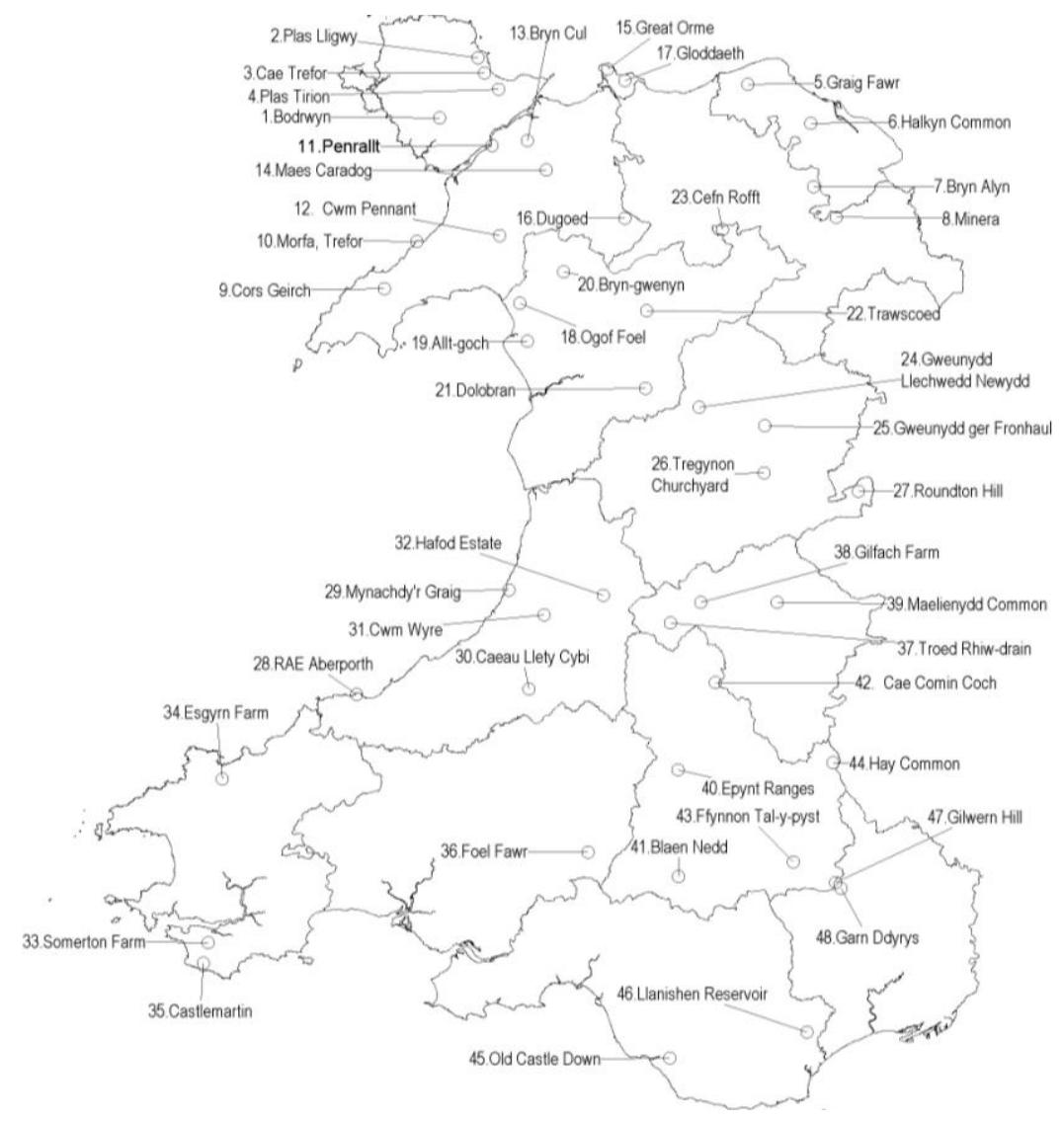

Fig. 1 - Map of Wales indicating the 48 sites sampled as part of this survey, showing vice-county boundaries. Site numbers correspond to those listed in Table 1. 


\section{Summary of taxa found}

A total of 35 species of Hygrocybe spp. (+4 subspecies), 46 of Entoloma spp. (+1 subspecies), 16 of Clavariaceae spp, 8 of Geoglossaceae, three of Camarophyllopsis spp., two of Dermoloma spp. and one of Porpoloma were found during the course of this survey giving a CHEGD total of 111 (16:35:46:8:6) (Table 2). These included one previously undescribed species (provisional name: Entoloma eryriensis), two new UK records (E. conocybecystis and E. glaucobasis) and additionally 12 new Welsh records. Thus, over 13\% (15/111) of the species recorded in this survey were new to Wales. Most (12/15) of the species new to Wales belonged to the genus Entoloma, indicating the extent of past under-recording of this genus in Wales. However, of the 46 Entoloma species, 16 were found at only a single site (Table 2). The most widespread species were Hygrocybe virginea and $H$. pratensis (46 sites), with a total of 10 species being found at $\geq 35$ sites. The notional UK maximum CHEGD score based on those grassland species listed by Legon \& Henrici (2005) and including the three new UK records would be 200 (21:51:106:12:10). Thus $56 \%$ of the possible grassland CHEGD taxa known for the UK were discovered at these 48 sites.

The mean number of CHEGD species found per site was 28.2 (range 3 to 55; combined data for both surveys; Table 1), with a mean of 19.4 species per site for individual surveys. The most abundant taxon was Hygrocybe (mean 14.6 [combined data] and 11.3 [individual survey] spp./site; range 1 to 25), followed by Entoloma (mean 5.8 [combined data] and 3.3 [individual survey] spp./site; range 0 to 24) with Clavariaceae, Geoglossaceae and 'Dermoloma' being present at lower levels (mean 4.6, 1.8 and $0.9 \mathrm{spp} . /$ site respectively [combined data]).

In order to identify sites of high grassland macrofungal diversity, various scoring systems have been devised (Rald 1985, Nitare 1988, Vesterholt et al. 1999, Rotheroe 2001) and widely used (Rotheroe 1999, Thompson 2000, McHugh et al. 2001, Newton et al. 2003). These systems aim to rank site according to their conservation value at international, national, regional and local levels, with Vesterholt (1999) employing the number of Hygrocybe spp. found during repeated or single site surveys. Based on the combined survey data Hygrocybe spp. found during our surveys, five sites (Table 1) would qualify as of international importance ( $\geq 22$ Hygrocybe spp.) and a further 15 as of national importance (17-21 Hygrocybe spp.). However, when assessing our individual survey data, 19 sites would rank as internationally important ( $\geq 15$ Hygrocybe spp.) and a further 15 as nationally important (11-14 Hygrocybe spp.). Using Nitare's (1988) classification for the other CHEGD taxa (based on counts for single visits), $\geq 6$ Clavariaceae were found at 16 sites, $\geq 9$ Entoloma spp. at 12 sites and $\geq 4$ Geoglossaceae at 10 sites. Additionally, one or more of the four CHEGD species for which Species Action Plans have been prepared as part of the UK Biodiversity Action Plan (E. bloxamii, H. spadicea, M. olivaeceum and T. atropurpureum) were found at 16 sites.

\section{Co-occurrence of different CHEGD taxa}

When combined survey data for sites were ranked according to the number of species of each taxon present (Table 1), the best 3 sites for Entoloma spp. ranked only $9^{\text {th }}, 17^{\text {th }}$ and $21^{\text {st }}$ for Hygrocybe spp., whilst the best three Hygrocybe sites ranked $5^{\text {th }}, 8^{\text {th }}$ and $13^{\text {th }}$ for Entoloma. Pearson linear correlation analysis based on individual surveys showed a moderate correlation between numbers of Clavariaceae and Hygrocybe (Table 3a), but weaker correlation for other pairwise combinations. A similarly poor correlation between the best sites for the different taxa (but based on single visits) was also found by Newton et al. (2003). 
Table 1 Summary of Survey Sites.

\begin{tabular}{|c|c|c|c|c|c|c|c|c|c|c|c|c|c|}
\hline Site name & VC & $\begin{array}{c}\text { OS } \\
\text { Gridref }\end{array}$ & $\begin{array}{c}{ }^{\dagger} \text { Site } \\
\text { Area } \\
\text { (ha) }\end{array}$ & $\begin{array}{l}{ }^{\jmath} \text { Phase I } \\
\text { Habitat }\end{array}$ & 2003 & 2004 & 2005 & ${ }^{\#} \mathbf{C}$ & $\#$ & ${ }^{\#} \mathbf{E}$ & ${ }^{\#} \mathbf{G}$ & ${ }^{\#} \mathbf{D}$ & $\begin{array}{c}{ }^{\#} \text { CHEGD } \\
\text { total }\end{array}$ \\
\hline 1 Bodrwyn $^{3}$ & 52 & SH415732 & $0.11^{P}$ & $\mathrm{NG}$ & $15-\mathrm{Nov}$ & & 9-Nov & 3 & 13 & 2 & 0 & 0 & 18 \\
\hline 2 Plas Lligwy $^{4}$ & 52 & SH496859 & $0.28^{\mathrm{P}}$ & NG & $27-\mathrm{Oct}$ & 4-Nov & & 2 & 12 & 2 & 2 & 1 & 19 \\
\hline 3 Cae Trefor ${ }^{3}$ & 52 & SH510827 & $0.22^{\mathrm{P}}$ & NG & 29-Oct & $4-\mathrm{Nov}$ & & 3 & 9 & 0 & 2 & 1 & 15 \\
\hline 4 Plas Tirion $^{4}$ & 52 & SH539793 & $2.2^{\mathrm{P}}$ & NG & $4-\mathrm{Nov}$ & 22-Sep & & 3 & 10 & 4 & 2 & 2 & 21 \\
\hline 5 Graig Fawr ${ }^{1 *}$ & 51 & SJ062803 & $24.5^{\mathrm{S}}$ & $\mathrm{CG}$ & $2-\mathrm{Oct}$ & 6-Nov & & 5 & 10 & 1 & 2 & 1 & 19 \\
\hline 6 Halkyn $^{1 *}$ & 51 & SJ194721 & $771.8^{\mathrm{S}}$ & CG & 3-Oct & 3-Nov & & 5 & 19 & 24 & 5 & 2 & 55 \\
\hline 7 Bryn Alyn ${ }^{1 *}$ & 50 & SJ200588 & $112.3^{\mathrm{S}}$ & CG & 1-Oct & $5-\mathrm{Nov}$ & & 4 & 16 & 16 & 5 & 1 & 42 \\
\hline 8 Minera $^{1 *}$ & 50 & SJ247525 & $43.7^{\mathrm{S}}$ & CG & $6-O c t$ & 7-Nov & & 3 & 17 & \begin{tabular}{|l|}
16 \\
\end{tabular} & 3 & 1 & 40 \\
\hline 9 Cors Geirch $^{3 *}$ & 49 & SH300374 & $224^{\mathrm{S}}$ & NG & 12-Nov & & 22-Oct & 4 & 5 & 2 & 0 & 1 & 12 \\
\hline 10 Morfa, Trefor $^{4}$ & 49 & SH368473 & $54.0^{\mathrm{P}}$ & SG & & 26-Oct & $16-$ Oct & 4 & 18 & 10 & 1 & 1 & 34 \\
\hline 11 Penrallt $^{4}$ & 49 & SH526674 & $0.27^{\mathrm{A}}$ & NG & 4-Dec & 18-Oct & & 4 & 17 & 2 & 0 & 1 & 24 \\
\hline 12 CwmPennant $^{1}$ & 49 & SH542486 & $56.6^{\mathrm{A}}$ & $A G$ & & 9-Oct & 24-Sep & 8 & 20 & 9 & 0 & 0 & 37 \\
\hline $13{\text { Bryn } \mathrm{Cul}^{3}}^{3}$ & 49 & SH600685 & $4.4^{\mathrm{P}}$ & NG & $11-\mathrm{Nov}$ & 16-Oct & & 6 & 18 & 3 & 0 & 1 & 28 \\
\hline 14 Maes Caradog ${ }^{3 *}$ & 49 & SH639623 & $5.9^{\mathrm{A}}$ & AG & 9-Nov & 9-Oct & & 5 & 16 & 6 & 1 & 0 & 28 \\
\hline 15 Great Orme $e^{2 *}$ & 49 & SH768835 & $353.3^{\mathrm{S}}$ & CG & 21-Nov & 26-Oct & & 7 & 18 & 8 & 3 & 1 & 37 \\
\hline 16 Dugoed $^{1 *}$ & 49 & SH804522 & $28.0^{\mathrm{A}}$ & $\mathrm{AG}$ & 30-Sep & 2-Nov & & 2 & 9 & 3 & 3 & 1 & 18 \\
\hline 17 Gloddaeth $^{1 *}$ & 49 & SH804811 & $93.1^{\mathrm{S}}$ & CG & 22-Nov & 7-Oct & & 4 & 18 & 8 & 0 & 1 & 31 \\
\hline 18 Ogof Foel $^{1}$ & 48 & SH583344 & $16.2^{\mathrm{P}}$ & $A G$ & & $24-O c t$ & 9-Oct & 5 & 20 & 12 & 0 & 1 & 38 \\
\hline 19 Allt-goch ${ }^{1}$ & 48 & SH599264 & $1.7^{\mathrm{P}}$ & UNG & & 17-Oct & 9-Oct & 6 & 15 & 11 & 2 & 2 & 36 \\
\hline 20 Bryn-gwenyn $^{1}$ & 48 & SH676410 & $10.1^{\mathrm{P}}$ & $A G$ & & 8-Nov & $17-$ Oct & 6 & 14 & 3 & 2 & 2 & 27 \\
\hline 21 Dolobran $^{4}$ & 48 & SH847165 & $131^{\mathrm{P}}$ & $\mathrm{AG}$ & & 9-Nov & 5-Oct & 4 & 17 & 4 & 2 & 3 & 30 \\
\hline 22 Trawscoed $^{2^{*}}$ & 48 & SH849328 & $418^{\mathrm{P}}$ & $\mathrm{AG}$ & & 30-Oct & 23-Sep & 2 & 24 & 8 & 1 & 0 & 35 \\
\hline 23 Cefn Rofft ${ }^{1 *}$ & 48 & SJ009498 & $4.1^{\mathrm{S}}$ & CG & 4-Oct & 2-Nov & & 3 & 12 & 4 & 0 & 1 & 20 \\
\hline $\begin{array}{l}24 \text { Gweunydd Llechwedd } \\
\text { Newydd }^{1 *}\end{array}$ & 47 & SH960126 & $3.5^{\mathrm{s}}$ & NG & 8 -Oct & 4-Nov & & 2 & 5 & 1 & 0 & 0 & 8 \\
\hline $\begin{array}{l}25 \text { Gweunydd ger } \\
\text { Fronhaul }^{1}\end{array}$ & 47 & SJ097087 & $5.5^{\mathrm{S}}$ & UNG & 8-Oct & 4-Nov & & 1 & 6 & 0 & 0 & 0 & 7 \\
\hline 26 Tregynon Churchyard ${ }^{4}$ & 47 & SO096988 & $0.18^{\mathrm{P}}$ & NG & 7-Oct & $4-\mathrm{Nov}$ & & 5 & 14 & 1 & 4 & 1 & 25 \\
\hline 27 Roundton Hill $^{1^{*}}$ & 47 & SO294949 & $37.7^{\mathrm{S}}$ & AG & & 4-Nov & $11-$ Oct & 6 & 12 & 2 & 3 & 1 & 24 \\
\hline 28 RAE Aberporth ${ }^{1 *}$ & 46 & SN242523 & $82.6^{\mathrm{P}}$ & UNG & & $6-\mathrm{Nov}$ & 13-Nov & 8 & 16 & 5 & 4 & 1 & 34 \\
\hline 29 Mynachdy'r Graig $^{4}$ & 46 & SN561743 & $62.1^{\mathrm{P}}$ & AG & & 24-Nov & 7-Oct & 8 & 15 & 3 & 2 & 0 & 28 \\
\hline 30 Caeau Llety Cybi ${ }^{1 *}$ & 46 & SN603535 & $3.7^{\mathrm{s}}$ & UNG & & $20-O c t$ & 20-Sep & 2 & 12 & 3 & 0 & 1 & 18 \\
\hline 31 Cwm Wyre $^{1}$ & 46 & SN635690 & $33.0^{\mathrm{P}}$ & UNG & & 23-Nov & 18 -Nov & 1 & 6 & 0 & 1 & 1 & 9 \\
\hline 32 Hafod Estate ${ }^{2 *}$ & 46 & SN759731 & $26.3^{\mathrm{A}}$ & NG & & 4-Nov & 23-Oct & 2 & 10 & 1 & 1 & 1 & 15 \\
\hline 33 Somerton Farm ${ }^{3}$ & 45 & SM930003 & $20.7^{\mathrm{P}}$ & NG & & 24-Oct & $16-$ Oct & 12 & 15 & 8 & 4 & 1 & 40 \\
\hline 34 Esgyrn Farm ${ }^{3}$ & 45 & SM960346 & $44.3^{\mathrm{P}}$ & NG & & 22-Oct & 14-Oct & 0 & 15 & 4 & 1 & 1 & 21 \\
\hline 35 Castlemartin $^{1 *}$ & 45 & SR920960 & $1878^{\mathrm{P}}$ & SG & & 23-Oct & 15-Oct & 4 & 11 & 6 & 0 & 1 & 22 \\
\hline 36 Foel fawr $^{3 *}$ & 44 & SN727193 & $77.2^{\mathrm{A}}$ & $\mathrm{CG}$ & & 4-Dec & 17-Oct & 0 & 3 & 0 & 0 & 0 & 3 \\
\hline 37 Troed Rhiw-drain ${ }^{1 *}$ & 43 & SN899673 & $19.6^{\mathrm{S}}$ & AG & & $14-\mathrm{Nov}$ & 8-Oct & 6 & 18 & 5 & 3 & 1 & 33 \\
\hline 38 Gilfach Farm ${ }^{2 *}$ & 43 & SN963716 & $84.7^{\mathrm{A}}$ & UNG & & $16-O c t$ & $10-\mathrm{Nov}$ & 9 & 22 & 7 & 4 & 1 & 43 \\
\hline 39 Maelienydd Common ${ }^{2 *}$ & 43 & SO123716 & $342^{\mathrm{S}}$ & $\mathrm{AG}$ & & $10-$ Oct & 22-Oct & 7 & 19 & 11 & 1 & 1 & 39 \\
\hline 40 Epynt Ranges ${ }^{2 *}$ & 42 & SN915365 & $14568^{\mathrm{P}}$ & $\mathrm{AG}$ & & 11-Nov & $18-$-Oct & 6 & 24 & 10 & 3 & 2 & 45 \\
\hline 41 Blaen Nedd ${ }^{1 *}$ & 42 & SN916142 & $187^{\mathrm{s}}$ & CG & & 7-Nov & $1-$-Oct & 6 & 25 & 11 & 4 & 1 & 47 \\
\hline 42 Cae Comin $\operatorname{Coch}^{2 *}$ & 42 & SN994548 & $1.3^{\mathrm{S}}$ & UNG & & 14-Nov & 8-Oct & 4 & 16 & 10 & 1 & 0 & 31 \\
\hline 43 Ffynnon Tal-y-pyst ${ }^{2}$ & 42 & SO157172 & $10.3^{\mathrm{A}}$ & $\mathrm{AG}$ & & 26-Oct & $4-\mathrm{Nov}$ & 2 & 18 & 5 & 3 & 1 & 29 \\
\hline 44 Hay Common $^{4}$ & 42 & SO240380 & $124.5^{\mathrm{A}}$ & AG & & 9-Oct & 6-Nov & 9 & 20 & 7 & 4 & 1 & 41 \\
\hline 45 Old Castle Down ${ }^{1 *}$ & 41 & SS898761 & $273.1^{\mathrm{S}}$ & SG & & 21-Nov & $16-$-Oct & 5 & 14 & 9 & 4 & 1 & 33 \\
\hline 46 Llanishen Reservoir ${ }^{4 *}$ & 41 & ST186815 & $6.7^{\mathrm{S}}$ & NG & & 20-Nov & 5-Nov & 4 & 15 & 6 & 0 & 1 & 26 \\
\hline 47 Gilwern Hill $^{1 *}$ & 35 & SO246129 & $81^{\mathrm{S}}$ & CG & & 26-Oct & 21-Oct & 10 & 23 & 4 & 4 & 0 & 41 \\
\hline 48 Garn Ddyrys ${ }^{2}$ & 35 & SO257117 & $4.3^{\mathrm{A}}$ & $\mathrm{CG}$ & 22-Oct & 25-Nov & & 4 & 19 & 3 & 1 & 0 & 27 \\
\hline
\end{tabular}

${ }^{\dagger}$ Site choice was based on 1) the results of the CCW Phase II vegetation survey of Lowland Grasslands in Wales; 2) sites with high grassland fungal diversity described in Evans \& Holden (2003); 3) identified as promising by CCW staff; or 4) identified by surveyors. * indicates sites partly or wholly noted as SSSIs. VC = Vice-County (Watsonian).

* Sites were defined by Property (ownership) ${ }^{\mathrm{P}}$, SSSI boundaries ${ }^{\mathrm{S}}$ or by arbitrary boundaries (defined by surveyors) ${ }^{\mathrm{A}}$.

${ }^{f}$ Phase I habitat types are listed for each site $(\mathrm{AG}=$ Acid grassland; $\mathrm{CG}=$ Calcicolous grassland; $\mathrm{NG}=\mathrm{Neutral}$ grassland; $\mathrm{SG}=$ Coastal grassland; $\mathrm{UNG}=$ Unimproved neutral grassland)

${ }^{\#}$ Species counts ranked $5^{\text {th }}$ or higher for each of the CHEGD group are indicated in shaded boxes 
Table 2 Frequency of occurrence of CHEGD species at sites and in quadrats, compared to number of UK records held in the FRDBI. **indicates new UK record; \# indicates new Welsh record; + indicates confirmed Welsh record

\begin{tabular}{|c|c|c|}
\hline Species & $\begin{array}{c}\text { No. sites } \\
(/ 48)\end{array}$ & $\begin{array}{l}\text { No. UK } \\
\text { records }\end{array}$ \\
\hline \multicolumn{3}{|l|}{$\overline{H Y G R O C Y B E(35+4)}$} \\
\hline H. aurantiosplendens & 9 & 298 \\
\hline H. calyptriformis & 22 & 1645 \\
\hline H. cantharellus & 11 & 849 \\
\hline H. ceracea & 33 & 2190 \\
\hline H. chlorophana & 34 & 4001 \\
\hline H. citrinovirens & 10 & 338 \\
\hline H. coccinea & 42 & 3486 \\
\hline H. colemannia & 9 & 413 \\
\hline H. conica & 38 & 5555 \\
\hline H. flavipes & 20 & 518 \\
\hline H. fornicata & 29 & 690 \\
\hline H. glutinipes & 19 & 732 \\
\hline H. helobia & 8 & 240 \\
\hline H. ingrata & 3 & 81 \\
\hline H. insipida & 41 & 2088 \\
\hline H. intermedia & 13 & 546 \\
\hline H. irrigata & 29 & 1709 \\
\hline H. lacmus & 2 & 243 \\
\hline H. laeta & 24 & 1813 \\
\hline H. marchii & 2 & 410 \\
\hline H. miniata & 9 & 1240 \\
\hline H. mucronella & 14 & 474 \\
\hline H. nitrata & 8 & 401 \\
\hline H. ovina & 5 & 255 \\
\hline H. persistens & 3 & 1215 \\
\hline H. pratensis & 46 & 4754 \\
\hline H. psittacina & 45 & 4726 \\
\hline H. punicea & 28 & 1965 \\
\hline H. quieta & 44 & 2045 \\
\hline H. reidii & 34 & 1808 \\
\hline H. russocoriacea & 22 & 1343 \\
\hline H. spadicea & 1 & 73 \\
\hline H. splendissima & 14 & 549 \\
\hline H. virginea & 46 & 5929 \\
\hline H. vitellina & 1 & 269 \\
\hline H. pratensis $v$. pallida & 6 & 518 \\
\hline H. psittacina $v$. perplexa & 6 & 124 \\
\hline H. virginea $v$ fuscescens & 8 & 220 \\
\hline H. virginea v. ochraceopallida & 15 & 362 \\
\hline \multicolumn{3}{|l|}{ CAMAROPHYLLOPSIS (3) } \\
\hline \#Cm. atropuncta & 2 & 87 \\
\hline Cm. foetens & 1 & 94 \\
\hline Cm. schulzeri & 3 & 60 \\
\hline \multicolumn{3}{|l|}{ DERMOLOMA (2) } \\
\hline D. cuneifolium & 35 & 1065 \\
\hline \#D. magicum & 2 & 5 \\
\hline \multicolumn{3}{|l|}{ PORPOLOMA (1) } \\
\hline P. metapodium & 1 & 151 \\
\hline \multicolumn{3}{|l|}{ ENTOLOMA (46+1) } \\
\hline E. ameides & 4 & 148 \\
\hline+ E. anatinum & 9 & 139 \\
\hline E. asprellum & 1 & 115 \\
\hline E. atroceruleum & 5 & 120 \\
\hline E. bloxamii & 7 & 225 \\
\hline E. caesiocinctum & 1 & 183 \\
\hline \#E. catalaunicum & 2 & 48 \\
\hline E. chalybaeum & 18 & 309 \\
\hline \#E. clandestinum & 4 & 70 \\
\hline E. conferendum & 37 & 2817 \\
\hline$* *$ E. conocybecystis & 1 & 0 \\
\hline E. corvinum & 8 & 284 \\
\hline **E. eryriensis sp. nov. & 1 & 0 \\
\hline E. exile & 10 & 226 \\
\hline
\end{tabular}

\begin{tabular}{|c|c|c|}
\hline Species & $\begin{array}{c}\text { No. sites } \\
(/ 48)\end{array}$ & $\begin{array}{l}\text { No. UK } \\
\text { records } \\
\end{array}$ \\
\hline $\begin{array}{l}\text { E. formosum } \\
* * \text { E. glaucobasis }\end{array}$ & $\begin{array}{l}5 \\
1\end{array}$ & $\begin{array}{c}267 \\
0\end{array}$ \\
\hline E. griseocyaneum & 6 & 266 \\
\hline E. hebes & 4 & 343 \\
\hline$+E$. hirtipes & 2 & 226 \\
\hline E. incanum & 3 & 300 \\
\hline E. infula & 9 & 205 \\
\hline E. jubatum & 1 & 320 \\
\hline E. lamprosus & 3 & 350 \\
\hline \#E. langei & 1 & 18 \\
\hline+ E. lividocyanulum & 3 & 110 \\
\hline \#E. longistriatum & 8 & 69 \\
\hline E. minutum & 1 & 57 \\
\hline \#E. nigroviolaceum & 2 & 93 \\
\hline \#E. ochromicaceum & 1 & 12 \\
\hline E. olorinum & 1 & 9 \\
\hline E. papillatum & 16 & 598 \\
\hline E. poliopus & 8 & 97 \\
\hline E. politum & 1 & 134 \\
\hline E. porphyrophaeum & 16 & 877 \\
\hline \#E. pratulense & 1 & 4 \\
\hline E. prunuloides & 15 & 482 \\
\hline \#E. pseudoturci & 1 & 27 \\
\hline E. roseum & 1 & 72 \\
\hline E. sericellum & 21 & 1419 \\
\hline E. sericeum & 16 & 1627 \\
\hline E. serrulatum & 14 & 948 \\
\hline E. sodale & 1 & 72 \\
\hline E. solstitiale & 1 & 36 \\
\hline E. turci & 3 & 102 \\
\hline E. undatum & 2 & 156 \\
\hline \#E. xanthochroum & 4 & 94 \\
\hline E. longistriatum v. sarcitulum & 1 & 171 \\
\hline \multicolumn{2}{|l|}{ CLAVARIA (7) } & 564 \\
\hline C. argillacea & 1 & 364 \\
\hline C. fragilis & 25 & 1129 \\
\hline C. fumosa & 20 & 641 \\
\hline C. incarnata & 2 & 102 \\
\hline C. straminea & 11 & 145 \\
\hline C. zollingeri & 5 & 229 \\
\hline \multicolumn{3}{|l|}{ CLAVULINOPSIS (7) } \\
\hline Cp. corniculata & 34 & 2002 \\
\hline Cp. fusiformis & 14 & 992 \\
\hline Cp. helvola & 38 & 2794 \\
\hline Cp. laeticolor & 21 & 427 \\
\hline Cp. luteoalba & 27 & 914 \\
\hline \#Cp. subtilis & 1 & 111 \\
\hline Cp. umbrinella & 6 & 244 \\
\hline \multicolumn{3}{|l|}{ RAMARIOPSIS (2) } \\
\hline R. biformis & 1 & 37 \\
\hline R. kunzei & 4 & 300 \\
\hline \multicolumn{3}{|l|}{ GEOGLOSSUM (5) } \\
\hline G. atropurpureum & 3 & 191 \\
\hline G. cookeanum & 6 & 590 \\
\hline G. fallax & 25 & 891 \\
\hline G. glutinosum & 15 & 426 \\
\hline G. umbratile & 7 & 347 \\
\hline \multicolumn{3}{|l|}{ MICROGLOSSUM (1) } \\
\hline \multicolumn{3}{|l|}{ TRICHOGLOSSUM (2) } \\
\hline T. hirsutum & 17 & 645 \\
\hline T. walteri & 3 & 76 \\
\hline
\end{tabular}


Table 3 Pearson correlation coefficients for the numbers of different CHEGD taxa found at each site (a) for individual surveys and (b) combined data from both surveys. Superscripts indicate the significance of the linear correlation as follows: *** $\mathrm{p}<0.001 ; * * \mathrm{p}<0.01 ;{ }^{*} \mathrm{p}<0.05$; ${ }^{\mathrm{NS}} \mathrm{P}>0.05$.

a).

\begin{tabular}{cccccc}
\hline & $\boldsymbol{C}$ & $\boldsymbol{H}$ & $\boldsymbol{E}$ & $\boldsymbol{G}$ & $\boldsymbol{D}$ \\
\hline $\mathbf{C}$ & - & & & & \\
$\mathbf{H}$ & $0.570^{* * *}$ & - & & & \\
$\mathbf{E}$ & $0.300^{* *}$ & $0.325^{* *}$ & - & & \\
$\mathbf{G}$ & $0.346^{* *}$ & $0.326^{* *}$ & $0.014^{\mathrm{NS}}$ & - & \\
$\mathbf{D}$ & $0.276^{* *}$ & $0.141^{\mathrm{NS}}$ & $0.193^{\mathrm{NS}}$ & $0.091^{\mathrm{NS}}$ & - \\
\hline
\end{tabular}

b).

\begin{tabular}{|c|c|c|c|c|}
\hline$C$ & $H$ & $E$ & $G$ & $D$ \\
\hline- & & & & \\
\hline $0.541 * * *$ & - & & & \\
\hline $0.284^{\mathrm{NS}}$ & $0.577 * * *$ & - & & \\
\hline $0.486 * * *$ & $0.371^{* *}$ & $0.410^{* *}$ & - & \\
\hline $0.069^{\mathrm{NS}}$ & $0.101^{\mathrm{NS}}$ & $0.252^{\mathrm{NS}}$ & $0.315^{*}$ & - \\
\hline
\end{tabular}

However, when linear correlation analysis was conducted with data combined from both surveys, a much stronger positive correlation $(\mathrm{p}<0.05)$ for Hygrocybe and Entoloma species richness was observed (Table 3b). This suggests that whilst Entoloma and Hygrocybe spp. tend to occur on the same sites, they do not always fruit at the same time. Further examination of phenological differences between these two taxa was conducted by plotting the dates of surveys against numbers of Hygrocybe and Entoloma species (Fig. 2) confirmed that whilst there was no significant correlation between survey data and Hygrocybe occurrence $(\mathrm{r}=0.044 ; \mathrm{P}=0.673)$, a significant negative correlation was observed for Entoloma $\operatorname{spp}(\mathrm{r}=-0.535 ; \mathrm{P}<0.001)$.

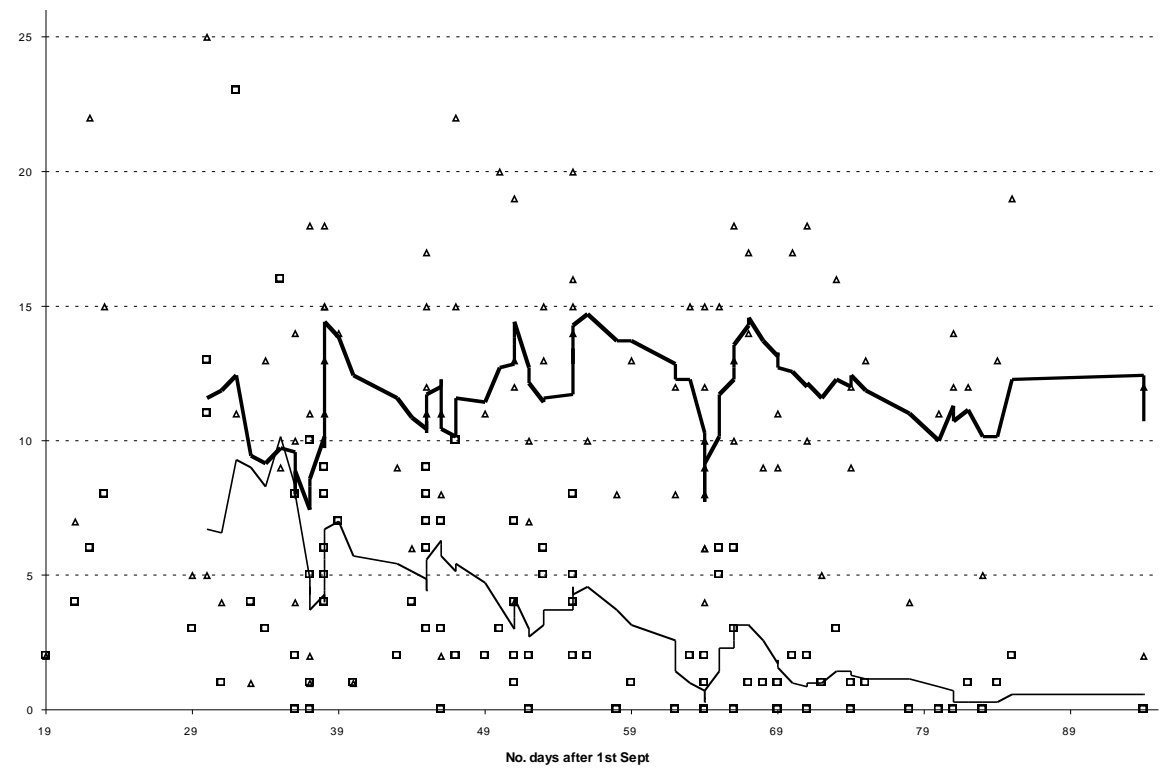

Fig. 2 - Numbers of Entoloma (squares; thin trendline) and Hygrocybe spp. (triangles thick trendline) recorded at sites plotted against survey date. The two trendlines indicate a moving window average over a 7 day period.

Fungal recording in the UK and Ireland is coordinated via the British Mycological Society (BMS) through the FRDBI. Although most records are not collected as part of systematic surveys, the large number of records held (>1 million) on the FRDBI make this an invaluable resource, with great potential for examining phenological variations. There are 95,585 records for CHEGD taxa (57,432 records for Hygrocybe, 20,887 for Entoloma, 11,511 for Clavariaceae, 4,130 for Geoglossaceae and together 1,625 for Dermoloma, Camarophyllopsis and Porpoloma). Analysis of the monthly patterns for records for CHEGD fungi found that most records for Entoloma are from September, whereas October is the peak month for Hygrocybe (Table 4), consistent with our survey data. 
Further examination of FRDBI data found a strong positive correlation between the total number of records for each species in the FRDBI and the occurrence of these species at sites $(\mathrm{r}=$ 0.851; Table 2). However, some outliers merit explanation, for instance Hygrocybe persistens (1215 FRDBI records, 333 in Wales) was found at only three sites in our surveys, probably because this species fruits earlier than other CHEGD taxa. Of the $H$. persistens records in the FRDBI with complete date information, the majority $(669 / 1144=58.4 \%)$ were recorded before October $1^{\text {st }}$, whilst only $17.7 \%$ [323/1822] and 24.4\% [1099/4499] respectively of $H$. punicea and $H$. psittacina records found before this date. Similarly, $H$. conica, which is the $2^{\text {nd }}$ most abundant Hygrocybe species in FRDBI, was ranked $7^{\text {th }}$ in our survey, is often found fruiting in the early summer with $40.2 \%(2126 / 5293)$ of FRDBI records occurring before $1^{\text {st }}$ October.

Table 4 Phenology of fruiting of CHEGD fungi by month in the British Isles (data extracted from FRDBI [http://www. fieldmycology.net]).

\begin{tabular}{|c|c|c|c|c|c|c|c|c|c|c|c|c|c|c|c|c|}
\hline & CHEGD & $\%$ CHEGD & CLAV & $\%$ CLAV & HYG & $\% \mathrm{Hyg}$ & ENT & $\%$ ENT & GEO & $\% \mathrm{GEO}$ & DERM & $\%$ DERM & Econf & $\%$ Econf & Hpsitt & $\%$ Hpsit \\
\hline Jan & 218 & $0.2 \%$ & 24 & $0.2 \%$ & 98 & $0.2 \%$ & 64 & $0.3 \%$ & 32 & $0.9 \%$ & 0 & $0.0 \%$ & 7 & $0.3 \%$ & 16 & $0.4 \%$ \\
\hline Feb & 68 & $0.1 \%$ & 6 & $0.1 \%$ & 10 & $0.0 \%$ & 49 & $0.3 \%$ & 3 & $0.1 \%$ & 0 & $0.0 \%$ & 13 & $0.5 \%$ & 6 & $0.1 \%$ \\
\hline Mar & 83 & $0.1 \%$ & 3 & $0.0 \%$ & 7 & $0.0 \%$ & 67 & $0.4 \%$ & 6 & $0.2 \%$ & 0 & $0.0 \%$ & 2 & $0.1 \%$ & 2 & $0.0 \%$ \\
\hline Apr & 220 & $0.2 \%$ & 7 & $0.1 \%$ & 28 & $0.1 \%$ & 182 & $1.0 \%$ & 3 & $0.1 \%$ & 0 & $0.0 \%$ & 25 & $0.9 \%$ & 5 & $0.1 \%$ \\
\hline May & 566 & $0.6 \%$ & 6 & $0.1 \%$ & 75 & $0.1 \%$ & 476 & $2.5 \%$ & 7 & $0.2 \%$ & 2 & $0.1 \%$ & 114 & $4.3 \%$ & 18 & $0.4 \%$ \\
\hline Jun & 539 & $0.6 \%$ & 26 & $0.2 \%$ & 252 & $0.5 \%$ & 256 & $1.4 \%$ & 4 & $0.1 \%$ & 1 & $0.1 \%$ & 55 & $2.1 \%$ & 10 & $0.2 \%$ \\
\hline Jul & 1175 & $1.3 \%$ & 42 & $0.4 \%$ & 582 & $1.1 \%$ & 542 & $2.9 \%$ & 1 & $0.0 \%$ & 8 & $0.5 \%$ & 59 & $2.2 \%$ & 19 & $0.4 \%$ \\
\hline Aug & 5512 & $6.2 \%$ & 288 & $2.7 \%$ & 2451 & $4.5 \%$ & 2599 & $13.8 \%$ & 96 & $2.6 \%$ & 78 & $5.2 \%$ & 346 & $13.1 \%$ & 120 & $2.7 \%$ \\
\hline Sep & 20570 & $23.1 \%$ & 2150 & $20.0 \%$ & 10929 & $20.2 \%$ & 6638 & $35.1 \%$ & 516 & $13.8 \%$ & 337 & $22.5 \%$ & 880 & $33.3 \%$ & 903 & $20.1 \%$ \\
\hline Oct & 39939 & $44.9 \%$ & 5597 & $52.0 \%$ & 25611 & $47.3 \%$ & 6203 & $32.8 \%$ & 1725 & $46.2 \%$ & 803 & $53.5 \%$ & 836 & $31.6 \%$ & 2147 & $47.7 \%$ \\
\hline Nov & 18609 & $20.9 \%$ & 2411 & $22.4 \%$ & 13087 & $24.2 \%$ & 1666 & $8.8 \%$ & 1191 & $31.9 \%$ & 254 & $16.9 \%$ & 291 & $11.0 \%$ & 1145 & $25.5 \%$ \\
\hline Dec & 1504 & $1.7 \%$ & 194 & $1.8 \%$ & 996 & $1.8 \%$ & 146 & $0.8 \%$ & 151 & $4.0 \%$ & 17 & $1.1 \%$ & 18 & $0.7 \%$ & 108 & $2.4 \%$ \\
\hline Total & 89003 & & 10754 & & 54126 & & 18888 & & 3735 & & 1500 & & 2646 & & 4499 & \\
\hline $\begin{array}{r}\% \text { Jan-Jun } \\
\text { records } \\
\end{array}$ & & $1.90 \%$ & & $0.67 \%$ & & $0.87 \%$ & & $5.79 \%$ & & $1.47 \%$ & & $0.20 \%$ & & $8.16 \%$ & & $1.27 \%$ \\
\hline
\end{tabular}

\section{Discussion}

The discovery of two species not previously found in the UK (one of which is new to science) and 12 new Welsh records during this study attests to the under-recording of grassland fungi in Wales. That most of these novel taxa were in the genus Entoloma is largely due to the complex taxonomy of this genus, reliant on the recognition of subtle morphological features for the identification of some species. Compared to the two earlier systematic surveys of grassland fungi (where the mean number of Hygrocybe spp. found at 474 Scottish sites surveyed by Newton et al. (2003) was 6.2 [including ca. 80 zero records] and for Thompson's (2000) surveys in Somerset with 7.4 Hygrocybe spp./site, the mean numbers of Hygrocybe species found in the surveys reported here were approximately 2 -fold higher, with only five $(10 \%)$ of the sites having fewer than nine species present. The reason for this is that sites were not selected at random but based on prior information that these sites were home to diverse populations of grassland macrofungi.

The 48 sites encompassed several grassland types but comparisons of CHEGD populations on acid, neutral and calcicolous grasslands did not reveal any significant differences in the numbers of species or the assemblages present (statistical analyses not shown). This is consistent with a study of Hygrocybe spp. conducted in Sweden by Öster (2008) who found little correlation between vegetation data and macrofungal diversity. This is an important issue in terms of practical conservation, since it demonstrates clearly that diverse fungal communities may occur in grasslands of unexceptional botanical diversity. A likely explanation for this lack of correlation is that plant community diversity is reduced under heavy grazing pressure (as found in most of upland Wales), whereas soil fungal communities are most sensitive to soil nutrient status (Griffith et al. 2004, Griffith \& Roderick 2008).

This study focused on the CHEGD fungi which comprise the majority of macrofungal species found in grassland habitats in Europe. However, the occurrence of non-CHEGD species was also noted, with over 120 species being recorded. Most were dung or dung-associated fungi 
(e.g. Panaeolus spp.), litter saprotrophs (e.g. Mycena spp.) or terricolous species (e.g. Agaricus spp.) (Griffith \& Roderick 2008). However, at all but one of the 14 calcareous and coastal grassland sites (CG and SG in Table 1), ectomycorrhizal species (10 species in total including several Cortinarius spp. [C. anomalus, C. cinnamomeus, C. croceus, C. pratensis and Tricholoma sulphureum var. hemisulphureum) were recorded in open grassland, usually in association with the shrub Helianthemum nummularium (Barden 2007). However at three sites, C. croceus and $C$. pratensis were recorded apparently in the absence of any woody host, consistent with the discovery by Harrington \& Mitchell (2002) that some Cortinarius spp. form mycorrhizal associations with non-woody hosts such as Carex. Informal observations by surveyors suggested that large populations of Cortinarius spp. were negatively correlated with high diversity/abundance of CHEGD fungi, but evidence to substantiate this possibility was not found here.

The sites surveyed here have largely escaped the effects of modern (late $20^{\text {th }}$ century) agriculture (including synthetic fertiliser addition and ploughing). However, some of the sites are known to have been disturbed in the past, for instance Llanishen Reservoir embankment (built in 1886) and Garn Ddyrys (ironworks constructed in 1817 but abandoned in 1860) (http://212.219.230.30/\$sitepreview/world-heritage-blaenavon.org.uk/whs-info/nomination/hills/ hilltram.htm). Examination of maps from Dudley Stamp's Land Use survey in the 1930s (Stamp 1937); http://www.visionofbritain.org.uk/maps/), showed that six of the sites comprised significant areas marked as arable (e.g. Aberporth, Caeau Llety Cybi, Cae Trefor), though there are no details of frequency of ploughing or other management. Based on these sites, the timescale for recovery of waxcap grasslands following soil disturbance would appear to be several decades.

Furthermore, there may be distinct successional processes which occur in soil following physical disturbance that are important for soil microbes (but which are distinct from above-ground processes). It is important to clarify that physical disturbance differs from the chemical disturbance caused by the addition of synthetic fertilizers, which is a recent $[<50$ yrs $]$ phenomenon, and potentially much more damaging. Given that other sites, in addition to those listed above, comprised areas that were probably ploughed or otherwise disturbed in the past, it is conceivable that CHEGD fungi may be more prolific on 'recovering' sites than at pristine undisturbed sites. For instance, the absence of the ectomycorrhizal shrub Helianthemum nummularium following a disturbance event may be beneficial to waxcaps and other CHEGD taxa.

Relative to its small land area $\left(20,779 \mathrm{~km}^{2} ;<7 \%\right.$ of the area covered by FRDBI [UK and Ireland]), there are an unusually high number of CHEGD records for Wales (ca, 27\% of the FRDBI total). However, in terms of land use, grassland is the dominant habitat in Wales (ca. 72\%), of which 30-40\% (mainly in upland areas) is classified as unimproved or semi-improved (Howe et al. 2005). Thus, the large areas of grassland in Wales (much of it heavily sheep-grazed), combined with its topography, making some areas less accessible to mechanised agriculture (i.e. ploughing and fertiliser application) in part explain the abundance of these fungi, relative to other parts of the UK and Europe. When comparing the 20 sites of highest grassland fungal species richness within the British Isles, Welsh sites account for the majority (Table 5), and comparison with data from continental Europe provides further evidence these high levels of species richness are unusual. For instance, the best site in Netherlands has 24 spp. (Arnolds 1995) and Slovakia 22 spp. (Adamik \& Kautmanova 2005), with Denmark, Sweden and Norway together having only four sites with more than 28 spp. (Boertmann (1995), JB Jordal, pers. comm.).

Using data for the total area of permanent pasture in Wales, Scotland and the UK $(13,830$, 48480 and $114,500 \mathrm{~km}^{2}$ respectively) to calculate FDRBI records of CHEGD fungi per $\mathrm{km}^{2}$ pasture (http://www.fao.org/ag/AGP/AGPC/doc/Counprof/britain/unitedkingdom.htm - 5pasture), the frequency of occurrence of the CHEGD species at the 48 sites surveyed here was plotted against the numbers of FRDBI records for each taxon for Wales, Scotland and UK (Figure 3). The negative binomial distribution model fitted to each of these three datasets illustrates the correlation of FRDBI data with patterns for these species at the 48 sites in Wales and also the greater density of CHEGD records for Wales compared to Scotland and the whole of the UK. It is perhaps surprising that the density of records is lower for Scotland than for the UK as a whole. We suggest that this 
Table 5 Top 20 Sites for Hygrocybe spp. and other CHEGD taxa in the British Isles.

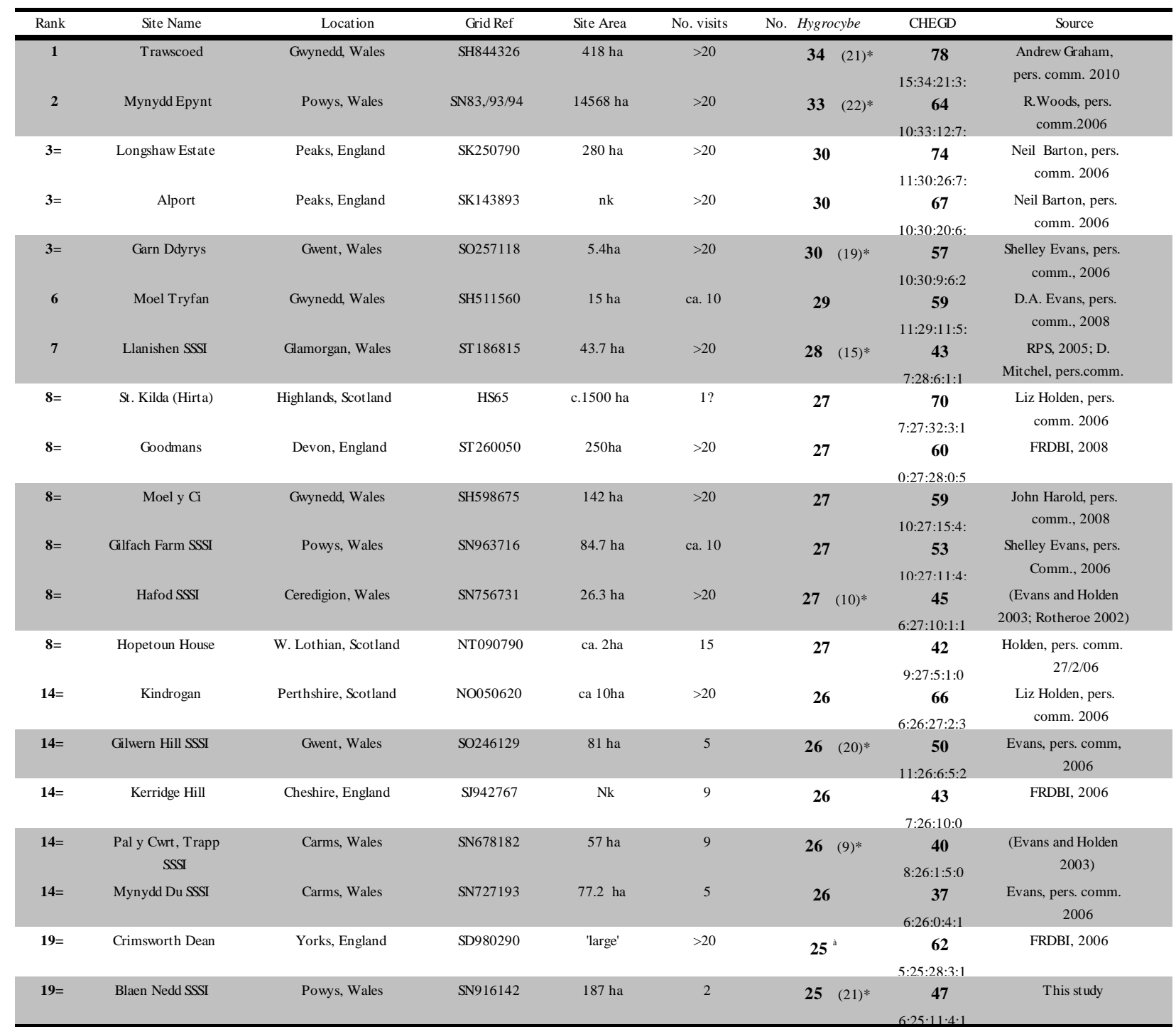

* Numbers in brackets indicate the highest count of Hygrocybe spp. in a single site survey during the present study. $\$$ Newton et al. (2003) reported 25 Hygrocybe spp. during a single visit at Rassal (Highlands). Shaded rows indicate sites in Wales. Taxa are counted according to the guidelines described in the methods section.

may partly be due to a lower intensity of surveying (fewer fungal recorders) but also reduced grazing pressure from sheep (ca. 8 million in both Wales and Scotland but the latter having a ca. 4fold greater land area).

Surprisingly 'waxcap grasslands' are virtually unknown outside northern Europe, presumably the result of factors such as recent glaciation, high rainfall levels and distinctive patterns of distribution of large mammalian herbivores linked to high human population densities. However, the CHEGD taxa have a worldwide distribution with many species found in Europe also found in boreal or tropical habitats (Halbwachs et al. 2013). For example Hygrocybe spp. are found in the Americas (Hesler \& Smith 1963, Pegler 1983), Africa (Heinemann 1963, Horak 1990) and Australasia (Horak 1990, Young 2006) but mostly in woodland habitats. The taxonomic treatment of these species does vary in other parts of the world (e.g generic names such as Gliophorus, Cuphophyllus [=Camarophyllus] and Neohygrocybe are applied to species known in Europe [sensu Boertmann] as Hygrocybe) and there are substantial differences in the species found in different parts of the world. For example, only 9/92 and 3/51 of the Hygrocybe species found in Australia and New Zealand respectively are also found in Europe, with high levels of endemism in both countries (e.g. 68/92 spp. endemic to Australia). There are occasional examples of Hygrocybe spp. 
in grassland habitats, such as on sand dunes in Australia (Young 2006), savannah in Central Africa (Heinemann 1963), coastal grasslands in California (Point Reyes; TD Bruns, pers. comm. 2006), as well as in churchyards in New Zealand (P Leonard, pers. comm. 2003). Furthermore, the presence of some Hygrocybe spp. have been revealed by genetic analyses in dry prairie soils (Lynch \& Thorn 2006) and semi-arid woodland (Zachow et al. 2009).

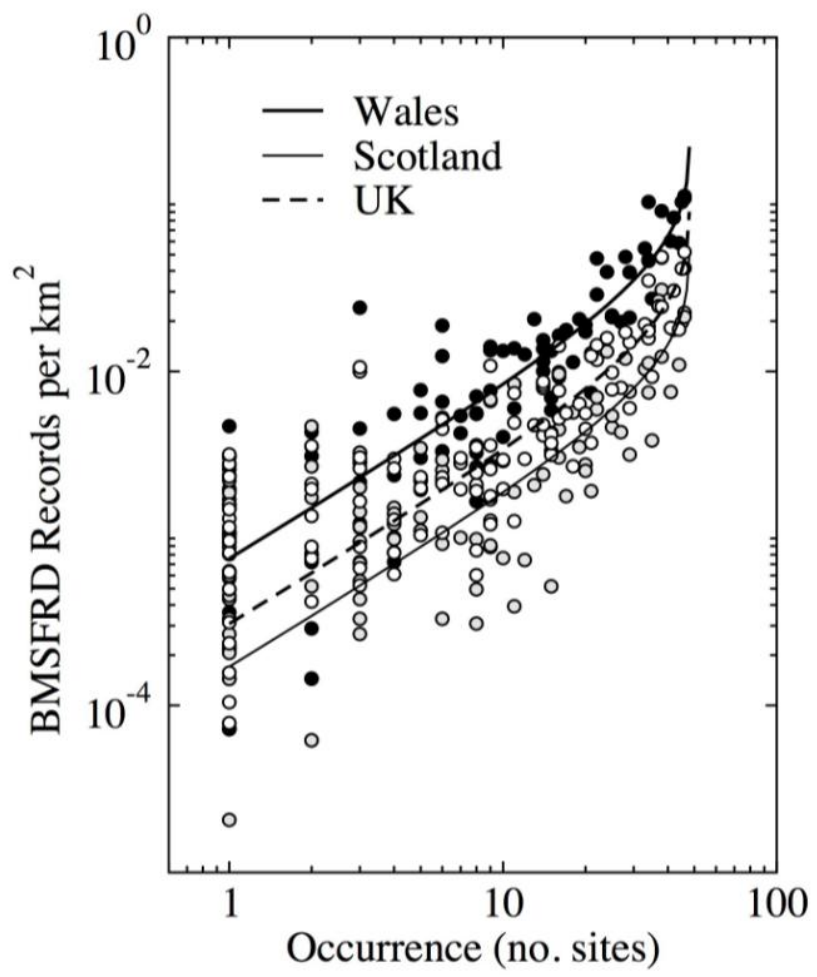

Fig. 3 - Number of FRDBI records for each species per $\mathrm{km}^{2}$ grassland area for Wales (black circles), Scotland (grey circles) and UK (open circles; excl. Republic of Ireland) plotted against the occurrence of these species at the 48 sites surveyed in this study. A negative binomial distribution model was used to fit the three curves to the three datasets. Grassland area was calculated using the data at http://www.fao.org/ag/AGP/AGPC/doc/Counprof/britain/unitedkingdom.htm - 5pasture.

Detailed surveying of sites for Hygrocybe spp. and the other CHEGD taxa at sites outside Europe has only rarely been conducted. However, Lane Cove Bushland Park, a sclerophyll woodland near the centre of Sydney has been listed in 2000 as a fungal heritage site under the Australian Heritage Commission Act, 1975 (http://www.sydneyfungalstudies.org.au/lanecove.htm) and $25 \mathrm{spp}$. are recorded for this $<1$ ha site. To our knowledge this is the only site outside the UK to have received legal protection due to its diverse Hygrocybe populations. Pegler (1983) reported that in Caribbean rainforest, Hygrocybe spp. are the most abundant of the macrofungi and several sites with $>20$ spp. of Hygrocybe (sensu Boertmann) have been reported in the Luquillo Mountains of Puerto Rico (http://dev.ites.upr.edu/data/luqmetadata86/7495). Similarly >30 Hygrocybe spp. have been recorded in the Great Smoky Mountain National Park in the US, with mossy areas in woodland glades having high species richness (JD Lodge, pers. comm. 2009). With fungal conservation lagging far behind most other groups of the biota, it is highly likely that more intensive surveying of other grassland/forest areas as we have conducted here will reveal areas of similar diversity.

Like many parts of North-Western Europe, where human activities over recent centuries have led to a significant loss of biodiversity, there are relatively few habitats in Wales whose conservation value is significant in an international context. Heavily-grazed grasslands such as those surveyed in the present study often have poor botanical (Olff \& Ritchie 1998) and animal diversity and are thus generally considered to be of low conservation value. It is thus pleasing that conservation bodies are beginning to account for the presence of diverse fungal populations (Senn- 
Irlet et al. 2007) and that the accumulation of data attesting to the high levels of macrofungal diversity in undisturbed pasture habitats will contribute to their protection by legislative means, including their formal integration into Agri-Environmental schemes. Furthermore, future changes in land-use, for instance afforestation for carbon sequestration, are likely to be targeted at low grade agricultural land, including upland pastures (e.g. Chapela et al. 2001). Thus we hope that our study along with parallel investigations of grassland fungi elsewhere in Europe and beyond, will bring to a wider audience the need to consider effects on grassland fungal populations if such land use changes are planned.

\section{Acknowledgements}

We dedicate this paper to the memory of our co-author David Stevens and also to Maurice Rotheroe, who in different ways provided the inspiration for this work but both have sadly died in the period since this study was conceived. We thank Eilir Evans, Jolanda Mitchel, Dave Bennett, Peter Roberts, Cory Mortis-Wait and Philip John for assistance with surveying. We are also grateful to Malcolm Storey, Kevin Roderick, Alick Henrici, John Warren, the late David Causton and Nigel Stringer for useful discussions and to Neil Barton, David Boertmann, John Bjarne Jordal and Jean Lodge for access to unpublished data. Finally we thank the Countryside Council for Wales for the funding of this work (contract no. FC-73-01-403) and to the many site owners for allowing access to these field sites. We also gratefully acknowledge funding for GWG/GLE under a Memorandum of Agreement project funded by CCW/SNH/NE/DoE NI/EHS/BMS/Plantlife and also to NERC.

\section{References}

Adamik S, Kautmanova I. 2005 - Hygrocybe species as indicators of natural value of grasslands in Slovakia. Catathelasma 6, 24-34.

Arnolds E. 1982 - Ecology and coenology of macrofungi in grasslands in Drenthe, the Netherlands. Vol. 2. Parts 2 \&3. Autecology and Taxonomy. Gantner Verlag K.G., Vaduz, Germany.

Arnolds E. 1988 - The Changing macromycete flora in the Netherlands. Transactions of the British Mycological Society 90(3), 391-406.

Arnolds E. 1992 - Macrofungal communities outside forests. In Winterhoff W. Fungi in Vegetation Science. - Kluwer Academic Publishers, Dordrecht, Netherlands, 113-150.

Arnolds E. 1995 - Conservation and management of natural populations of edible fungi. Canadian Journal of Botany 73, S987-S998.

Arnolds E, de Vries B. 1989 - Oecologische statistiek van de Nederlandse macrofungi. Coolia 32, 72-86.

Barden N. 2007 - Helianthemum grasslands of the Peak District and their possible mycorrhizal associates. Field Mycology 8(4), 119-126.

Bardgett RD, McAlister E. 1999 - The measurement of soil fungal : bacterial biomass ratios as an indicator of ecosystem self-regulation in temperate meadow grasslands. Biology and Fertility of Soils 29(3), 282-290.

Benton TG, Bryant DM, Cole L, Crick HQP. 2002 - Linking agricultural practice to insect and bird populations: a historical study over three decades. Journal of Applied Ecology 39, 673-687.

Boertmann D. 1995 - The genus Hygrocybe. Danish Mycological Society.

Cannon PF. 1997 - Strategies for rapid assessment of biodiversity. Biodiversity and Conservation 6, 669-680.

Chapela IH, Osher LJ, Horton TR, Henn MR. 2001 - Ectomycorrhizal fungi introduced with exotic pine plantations induce soil carbon depletion. Soil Biology \& Biochemistry 33(12-13), $1733-1740$.

Davies H. 1813 - Welsh Botanology; Part of the first. A systematic catalogue of the native plants of the Isle of Anglesey in Latin, English and Welsh (with the habitats of the rarer species and a few observations). W. Marchant London (Available fromhttp://books.google.co.uk). 
Evans SE, Holden EM. 2003 - Collation of data and information on mycologically important seminatural grasslands in Wales. . Report to Countryside Council for Wales. Bangor.

Feest A. 2000 - The assessment of the fungal value of sites for conservation. The Mycologist 14(1), 14-15.

Flynn DFB, Gogol-Prokurat M, Nogeire T, Molinari N, Richers BT, Lin BB, Simpson N, Mayfield MM, DeClerck F. 2009 - Loss of functional diversity under land use intensification across multiple taxa. Ecology Letters 12, 22-33.

Griffith GW, Bratton JL, Easton GL. 2004 - Charismatic megafungi: the conservation of waxcap grasslands. British Wildlife 15(3), 31-43.

Griffith GW, Easton GL, Jones AW. 2002 - Ecology and diversity of waxcap (Hygrocybe spp.) fungi. Botanical Journal of Scotland 54(1), 7-22.

Griffith GW, Roderick K. 2008 - Saprotrophic basidiomycetes in grasslands: distribution and function. In Boddy L, Frankland JC, van West P. Ecology of Saprotrophic Basidiomycetes. British Mycological Society Symposia Series. - Elsevier Ltd., London, 277-299.

Halbwachs H, Karasch P, Griffith GW. 2013 - The diverse habitats of Hygrocybe - peeking into an enigmatic lifestyle. Mycosphere in press.

Harrington TJ, Mitchell DT. 2002 - Colonization of root systems of Carex flacca and C. pilulifera by Cortinarius (Dermocybe) cinnamomeus. Mycological Research 106, 452-459.

Heinemann P. 1963 - Champignons recoltes au Congo par Madame M. Goossens-Fontana V. Hygrophoraceae. Bulletin du Jardin Botanique l'Etat a Bruxelles 33(4), 421-458.

Hesler LR, Smith AH. 1963 - North American Species of Hygrophoraceae. University of Tennessee Press, Knoxville, Tennessee.

Hewins EJ, Pinches C, Arnold J, Lush M, Robertson H, Escott S, 2005 - The condition of lowland BAP priority grasslands: results from a sample survey of non-statutory stands in England. English Nature Research Report 636. English Nature.

Horak E. 1990 - Monograph of the New-Zealand Hygrophoraceae (Agaricales). New Zealand Journal of Botany 28(3), 255-309.

Howe L, Blackstock T, Burrows C, Stevens J. 2005 - The habitat survey of Wales. British Wildlife February 2005, 153-162.

Jordal JB. 1997 - Sopp i naturbeitemarker i Norge. En kunnskapsstatus over utbredelse, okologi, indikatorverdi og trusler i et europeisk perspektiv. Utredning for DN 1997-6. Trondheim: Direktoratet for naturforvaltning.

Legon NW, Henrici A. 2005 - Checklist of the British and Irish Basidiomycota. RBG Kew, London.

Lodge DJ, Matheny PB, Cantrell SA, Moncalvo J-M, Vilgalys R, Redhead S. 2006 - Delineating the Hygrophoraceae: Waxy Myth vs. Gene Trees. Poster presented at Mycological Society of America Meeting, Québec, Canada. Available from http://www.aber.ac.uk/waxcap/downloads/Lodge2006-HygrophoraceaeMSAposter.pdf.

Lovegrove R, Shrubb M, Williams I. 1995 - Silent Fields, Royal Society for the Protection of Birds (Wales).

Lynch MD, Thorn RG. 2006 - Diversity of basidiomycetes in Michigan agricultural soils. Applied and Environmental Microbiology 72(11), 7050--7056.

McHugh R, Mitchel D, Wright M, Anderson R. 2001 - The fungi of Irish grasslands and their value for nature conservation. Biology and Environment: Proceedings of the Royal Irish Academy 101B(3), 225-242.

Newton AC, Davy LM, Holden L, Silverside A, Watling R, Ward SD. 2003 - Status, distribution and definition of mycologically important grasslands in Scotland. Biological Conservation $111,11-23$.

Nitare J. 1988 - Jordtungor, en svampgrupp pa tillbakagang i naturligafodermarker. Svensk Botanisk Tidskrift 82, 341-368.

Noordeloos ME. 1992 - Entoloma, s.l. (Fungi Europaei 5). Libreria editrice Giovanna Biella, Saronno, Italy. 
Noordeloos ME. 2004 - Entoloma s.1. Supplemento. Massimo Candusso, Alassio SV, Italy.

Olff H, Ritchie ME. 1998 - Effects of herbivores on grassland plant diversity. Trends in Ecology \& Evolution 13(7), 261-265.

Öster M. 2008 - Low congruence between the diversity of waxcaps (Hygrocybe spp.) fungi and vascular plants in semi-natural grasslands. Basic and Applied Ecology 9, 514-522.

Pegler DN. 1983 - Agaric Flora of the Lesser Antilles. HMSO, London, UK.

Rald E. 1985 - Vokshatte som indikatorarter for mykologisk vaerdifulde overdrevslokaliteter. Svampe 11, 1-9.

Ridge I. 2006 - Beginners Guide to Earth Tongues. North-West Fungus Group (NWFG) Newsletter (ISSN 1465-8054) June, http://fungus.org.uk/nwfg/earth-tongues.htm.

Rosenzweig M. 1995 - Species Diversity in Space and Time. Cambridge University Press.

Rotheroe M. 1999 - Mycological survey of selected semi-natural grasslands in Carmarthenshire. Contract Science Report No.340. Bangor: Countryside Council for Wales.

Rotheroe M. 2001 - A preliminary survey of waxcap grassland indicator species in South Wales. In Moore D, Nauta MM, Evans SE, Rotheroe M. Fungal Conservation: Issues and Solutions. Cambridge University Press, Cambridge, U.K., 120-135.

Rotheroe M, Newton A, Evans SE, Feehan J. 1996 - Waxcap-grassland survey. The Mycologist $10,23-25$.

Senn-Irlet B, Heilmann-Clausen J, Genney D, Dahlberg A. 2007 - Guidance for conservation of macrofungi in Europe. Document prepared for the Directorate of Culture and Natural Heritage Council of Europe, Strasbourg, October 2007.

Silverside AJ. 1997 - Keys to the Geoglossaceae (unpublished).

Stamp LD. 1937 - The Land of Britain. The Report of the Land Utilisation Survey of Britain.

Stevens DP, Blackstock TH, Smith SLN, Bosanquet SDS. 2007 - Lowland grassland survey of Wales. British Wildlife 18 (5 (June 2007)), 314-323.

Stevens DP, Smith SLN, Blackstock TH, Bosanquet SDS, Stevens JP. 2009 - Grasslands of Wales: a survey of lowland species-rich grasslands, 1987-2004. . University of Wales Press, Cardiff.

Straatsma G, Ayer F, Egli S. 2001 - Species richness, abundance and phenology of fungal fruit bodies over 21 years in a Swiss forest plot. Mycological Research 105(5), 515-523.

Thompson R, 2000 - The Somerset Grassland Fungi Project 1997-1999. Final Report, Somerset Environmental Records Centre.

Vesterholt J. 2002 - Contribution to the knowledge of species of Entoloma subgenus Leptonia growing in dry grassland. Massimo Candusso (ISSN-1128-6008), Alassio SV, Italy.

Vesterholt J, Boertmann D, Tranberg H. 1999 - et usaedvanlig godt ar for overdrevssvampe. Svampe 40, 36-44.

Young AM. 2006 - Fungi of Australia: Hygrophoraceae. CSIRO Publishing, 179 pp. ISBN: 0643091955.

Zachow C, Berg C, Muller H, Meincke R, Komon-Zelazowska M, Druzhinina IS, Kubicek CP, Berg G. 2009 - Fungal diversity in the rhizosphere of endemic plant species of Tenerife (Canary Islands): relationship to vegetation zones and environmental factors. ISME Journal 3, 79-92. 